\title{
The Synthesis of Dwell Mechanisms on the Basis of Straight-Line Linkages with Fivefold Interpolation Nodes
}

\author{
Viacheslav Kharzhevskyi ${ }^{1}$, Mykhaylo Pashechko ${ }^{2 *}$, Oleksandr Tatsenko ${ }^{3}$, \\ Maksym Marchenko', Pavlo Nosko ${ }^{4}$
}

1 Department of Industrial and Agricultural Engineering, Khmelnytskyi National University, 11 Instytutska str., 29016, Khmelnytskyi, Ukraine

2 Department of Fundamentals of Technology, Lublin University of Technology, 38A Nadbystrzycka str., 20-618 Lublin, Poland

3 Department of Operation of Machinery, Sumy National Agrarian University, 160 Kondratieva str., 40000, Sumy, Ukraine

4 Department of Mechanical Engineering, National Aviation University, 1, Komarova Ave.,02000, Kyiv, Ukraine

* Corresponding author's email: mpashechko@hotmail.com

\begin{abstract}
The paper deals with the problem of kinematic synthesis of dwell linkage mechanisms which are based on the four-bar linkage with a coupler point that traces a curve with a straight-line part. It enables to obtain a dwell of the output link of the mechanism, but the main goal is to find such parameters of the mechanisms that hold the required conditions of the designer: necessary dwell time, to satisfy the necessary cyclogram of the machine with taking into account the number of additional parameters. The main problem is to carry out the kinematic synthesis of the basic straight-line linkage that can be done by the kinematic geometry methods. In this paper it was shown that the fivefold interpolation nodes of the coupler curve with the tangent line can be successfully used to satisfy the necessary criteria. As a result of the carried out studies, the regions of existence of such mechanism parameters were determined enabling to carry out an optimization procedure using different criteria.
\end{abstract}

Keywords: straight-line linkages, kinematic geometry, dwell mechanisms, fivefold interpolation nodes, optimization synthesis.

\section{INTRODUCTION}

The problem of the optimal kinematic synthesis of mechanisms is one of the most complicated parts during the whole design process of machines. In many cases it is an important task to synthesize a mechanism which actuator has to move along a straight line or has a dwell with a prescribed duration according to the cyclogram of the machine. During that dwell, a certain technological operation can be performed. For example, in a raschel machine - thread is laid, in a packing machine - label is attached, in a double-action press - a part is pressed $[2,5,9]$. As shown in the paper [1], straight-line linkage mechanisms can be also used as building elements of small precise robotic devices.

Although the mechanisms with higher kinematic pairs are often used (cam mechanisms, Geneva-type mechanisms, etc.), it is known that linkage mechanisms are more durable and reliable due to the geometric closure of the links and absence of the higher kinematic pairs. However, the main problem of the linkage mechanisms usage is their complicated kinematic synthesis, the known methods usually allow obtaining just partial and not always the best solution [6,7].

It is known that dwell linkage mechanisms can be synthesized by means of the kinematic geometry methods of infinitesimally close positions 
of the plane figure [3,9]. The synthesis of path generating linkage mechanisms (circular and straight-line) by means of the afore-mentioned methods are based on the usage of special points of the coupler plane usage for its certain positions. Thus, a Burmester's points can be used to synthesize a mechanism with approximation of the coupler curve's part to the arc, as shown in the paper [4], and Ball's points can be used to synthesize straight-line linkages $[3,8]$. The partial case when those points are coincident at the certain position of the mechanism (a Chebyshev's or Ball-Burmester's point) was described in the work [9]. The afore-mentioned special point is the fivefold interpolation node and it can be used for designing the straight-line linkages and dwell mechanisms of their basis, and the main advantage is that the theoretical accuracy of the approximation is higher in comparison to other methods. However, the main problem is that the regions of existence of those mechanism parameters have not been determined yet, and it is also an important task to allow the designer to carry out the synthesis procedure of a mechanism by given dwell time and maximum displacement of the actuator, possible sizes of the links, conditions of forces transmission and other parameters. Thus, the goal of the paper was to determine the possible design parameters (regions of existence) of the dwell mechanisms that are built on the basis of straight-line linkages with the fivefold interpolation nodes of the coupler curve, in order to carry out their optimal kinematic synthesis by different criteria.

\section{DEFINITION OF THE SPECIAL POINTS OF THE COUPLER PLANE}

Figure 1 shows the kinematic scheme of the straight-line four-bar linkage that was synthesized by means of kinematic geometry methods. The mechanism includes the crank $r=l_{O A}$, the coupler $b=l_{A B}$ and the rocker $c=l_{B C}$. The length of all links is relative according to the distance between the fixed joints $O$ and $C: d=1$. The mechanism operates as follows: during the continuous rotation of the crank $O A$, the coupler point $T$ traces a path (coupler curve 5). The task is to correctly choose the position of the coupler point $T$ that is defined by the second arm of the coupler $k=l_{B T}$ and angle $\Omega_{T}$, taking into account the parameters of mechanism $r, b, c$, that enables point $T$ to draw a coupler curve with a straight line part $D_{1} D_{2}$. Thus, every point of the coupler plane $A B T$ of the mechanism can be chosen as a coupler point. In order to synthesize a path generating mechanism, the main idea of the kinematic geometry methods is to choose one of the special points that provides a high order of tangency of the coupler curve to its tangent line or circle in this point.

As the crank $O A$ rotates on the angle $\varphi_{1}$, the position of the mechanism links and the coupler point $T$ also changes. In the kinematic geometry theory, the special points of the coupler plane of the mechanism can be determined for any position of the mechanism. For example, if we change the crank $O A$ position that is defined by $\varphi_{1}$ angle, by the step of 1 degree, we can theoretically obtain 360 different path generating mechanisms with the different dimensions of the links, coupler curves, and thus parameters of the approximated parts. As it is known $[3,8,9]$, the Ball's points $U$ can be used to design a straight-line linkage. They provide $3^{\text {rd }}$ order of tangency between the coupler curve and the tangent line in this point and is characterized by the following condition as:

$$
K=d K / d \varphi_{1}=0,
$$

where: $K$ - the curvature of the coupler curve at this point. And as shown in the paper [4], Burmester points $S_{1}, S_{2}$ can be used for the synthesis of circular path generating mechanisms with the $4^{\text {th }}$ order of tangency. These points hold the following condition:

$$
K=\frac{1}{R} \neq 0 ; \frac{d K}{d \varphi_{1}}=\frac{d^{2} K}{d \varphi_{1}^{2}}=0,
$$

where: $R$ is the radius of a circle that approximates a part of the coupler curve.

If to define the Ball's points and Burmester points for every position of the mechanism, we can find the locus of those points for the basic four-bar linkage that is defined by the length $r, b, c$. The method of defining Burmester points was considered in the paper [4], and the Ball's points can be determined by using the methods that are shown in the work [9]. By using the mentioned methods, it is possible to draw a Ball's curve and Burmester points curve (the locus of appropriate points for the defined four-bar linkage). The sample of the obtained curves that were calculated and drawn by the developed software, are shown in Figure 2. 


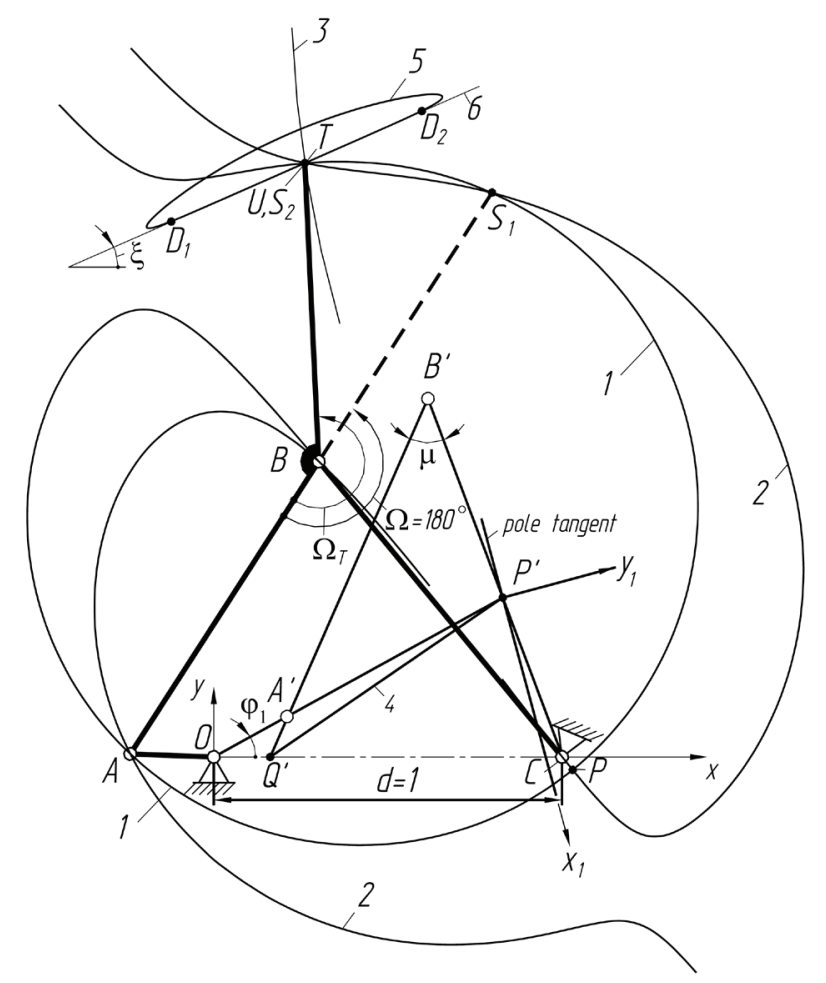

Fig. 1. Straight-line four-bar linkage $O A B C T$ that is synthesized by kinematic geometry methods:

1 - circle point curve; 2 - locus of the points with $4^{\text {th }}$ order of tangency; 3 - arc of the inflection circle; 4 - collineation axis; 5 - coupler curve with approximated part $D_{1} D_{2} ; 6$-straight line. Special points: $S_{1}-$ the first Burmester point, $T$ - the Ball-Burmester point (fivefold interpolation node), coincides with the Ball point $U$ and the second Burmester point $S_{2}$.

If in condition (2) we have the case where $K=0$, then the conditions (1) and (2) defines a partial case that is called a Ball-Burmester point (fivefold interpolation node): in that case the mentioned points coincide and the obtained straight-line mechanism has higher order of approximation of the coupler curve $\left(4^{\text {th }}\right.$ order of tangency instead of $3^{\text {rd }}$ ) and thus less deviation from the straight line on the approximated part of the coupler curve. Thus, the intersection points of the curves 1 and 2 (Fig. 2) can define a Ball-Burmester point, in the case where the coupler plane positions that are defined by the $\varphi_{1}$ angle are also equal.

In Figure 2, the graphical interpretation of the fivefold interpolation nodes (Ball-Burmester points) can be seen, but it is more convenient to find them analytically. Thus, since these points are defined as intersection of three curves (1,2 and 3 - see Fig. 1), a system of equations of those curves can be written [3], but such system will be overconstrained, because 3 curves in general case do not intersect in one point. Therefore, for the determination of the fivefold interpolation nodes the authors used a numerical and analytical method that is based on the following known statement of the kinematic geometry: if in the certain position of the coupler plane three Burmester points lie on the same line, then the $4^{\text {th }}$ Burmester point is a Ball-Burmester point (fivefold interpolation node). It should be noted that the moving joints $A$ and $B$ of the four-bar mechanism $O A B C T$ (see Fig.1) can also be considered as Burmester points, because they draw ideal circles and in general case have the tangency not only the $4^{\text {th }}$, but any higher order. Therefore, the following statement can be written: for the case when three Burmester points lie on the same line, it is sufficient to find such a position of the coupler plane $A B T$ of the mechanism where the Burmester point $S_{1}$ defines a mechanism with the coupler angle $\Omega=180^{\circ}$ (see Fig. 1). In that case, another Burmester point $S_{2}$, that can be found at the same position of the mechanism which is defined by the angle $\varphi_{1}$, will be a Ball-Burmester point (fivefold interpolation node). Thus, the appropriate algorithm and software were created and the mentioned special 


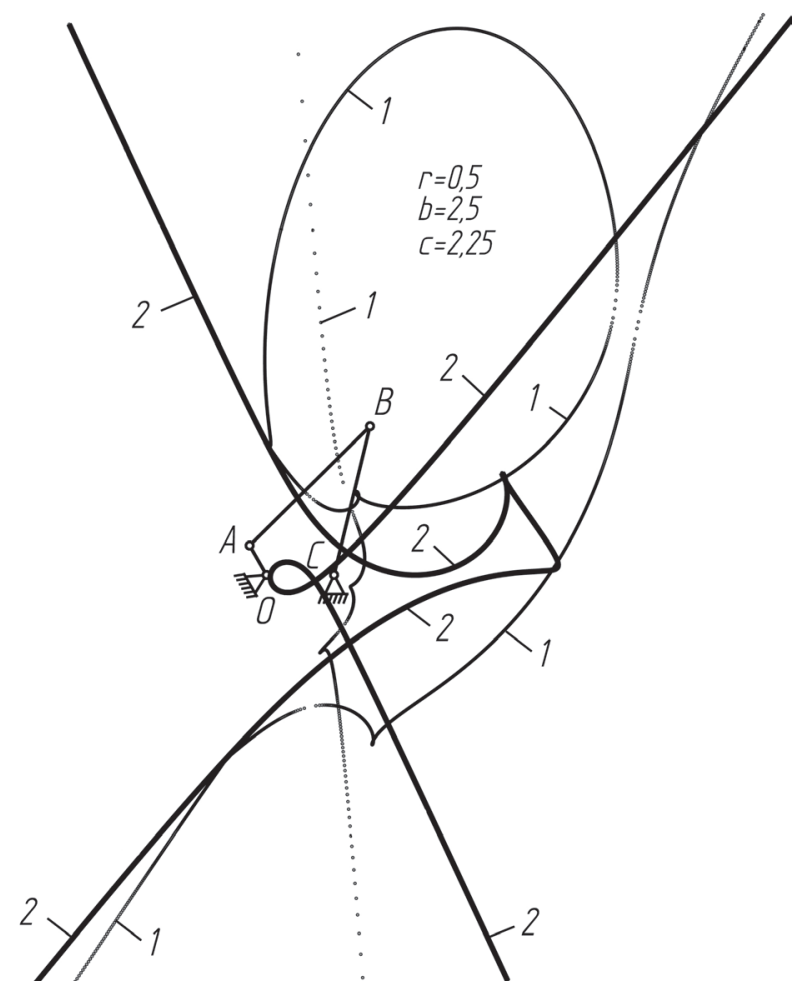

Fig. 2. Samples of the Burmester points curve (1) and Ball's curve (2) for the basic four-bar linkage

points were found numerically, as the points that lie on the Burmester points curve (see Fig. 2). Using that method, it was established for the first time that the locus of the Ball-Burmester points are also the curves that can be built for the given values of lengths of coupler $b$ and rocker $c$ of the mechanism from the minimum to the maximum values of a crank $r$. It should be also noticed that the range of $r$ values can be determined taking into account the Grashof condition of crank $r$ existence. In order to illustrate the obtained results, the examples of Ball-Burmester points' curves that were built for the different combinations of $r, b, c$ are shown in the Fig. 3. Obviously, the developed software allows finding the appropriate points for any initial parameters of the mechanisms.

Every point of the Ball-Burmester points' curves (Fig. 3) defines a straight-line mechanism, for which it is possible to find the length of the other arm of the coupler $k=l_{B T}$ and appropriate angle of the coupler $\Omega$ (Fig. 1). By the usage of the afore-mentioned numerical and analytical methods for the determination of the fivefold interpolation nodes, these parameters can be also determined. Fig. 4 shows the samples of appropriate diagrams.

\section{THE EXISTENCE REGIONS OF PARAMETERS OF DWELL MECHANISMS}

On the basis of the four-bar path generating linkage mechanism (see Fig. 1) it is possible to design a 6-link dwell mechanism that is shown in Figure 5. It consists of the basic four-bar linkage $O A B C D$ and the joined structural group $D E$ of the II class and $5^{\text {th }}$ type: during the continuous rotation of the crank $r=l_{O A}$, the coupler point $D$ traces a curve that has a straight-line part $D^{\prime} D^{\prime \prime}$. The output link $E$ (actuator) of the mechanism has a dwell during the time when the slider $D$ goes through the straight-line part of the coupler curve. As a crank rotates uniformly, the dwell time can be expressed as an angle $\alpha_{\Sigma}$ of the crank rotation during the dwell period. As it was established, for the certain basic four-bar linkage $O A B C$, two Ball-Burmester points $T_{1}$ and $T_{2}$ can be found in many cases (Fig. 5), but it is also possible to find 4,6 or 0 , each of them can generate a coupler curve with a straight-line part.

The important practical task during the design process of machines is the synthesis of linkage mechanisms by the given duration of the dwell of the output link. Several works in the linkage synthesis by means of fivefold interpolation 
a)

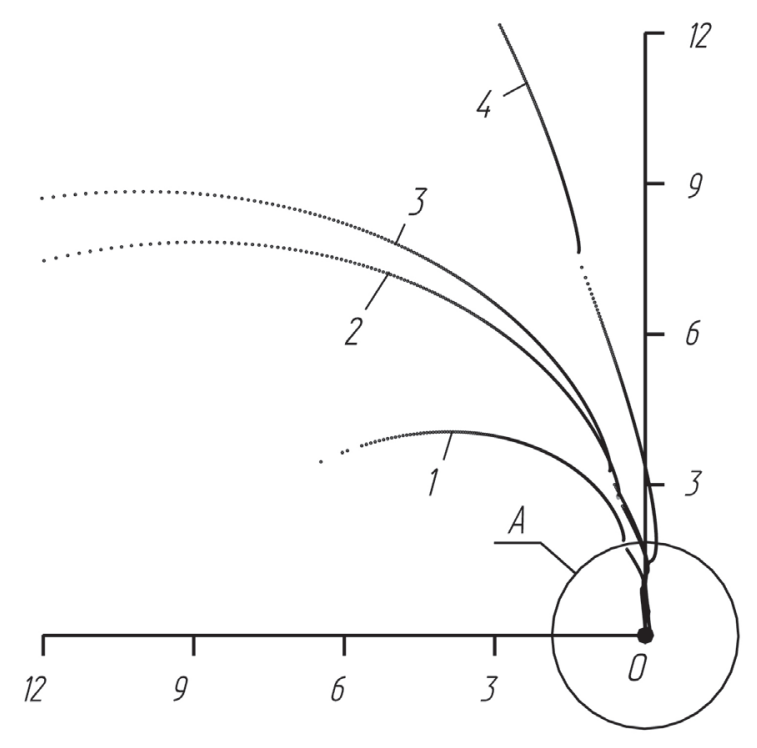

b)

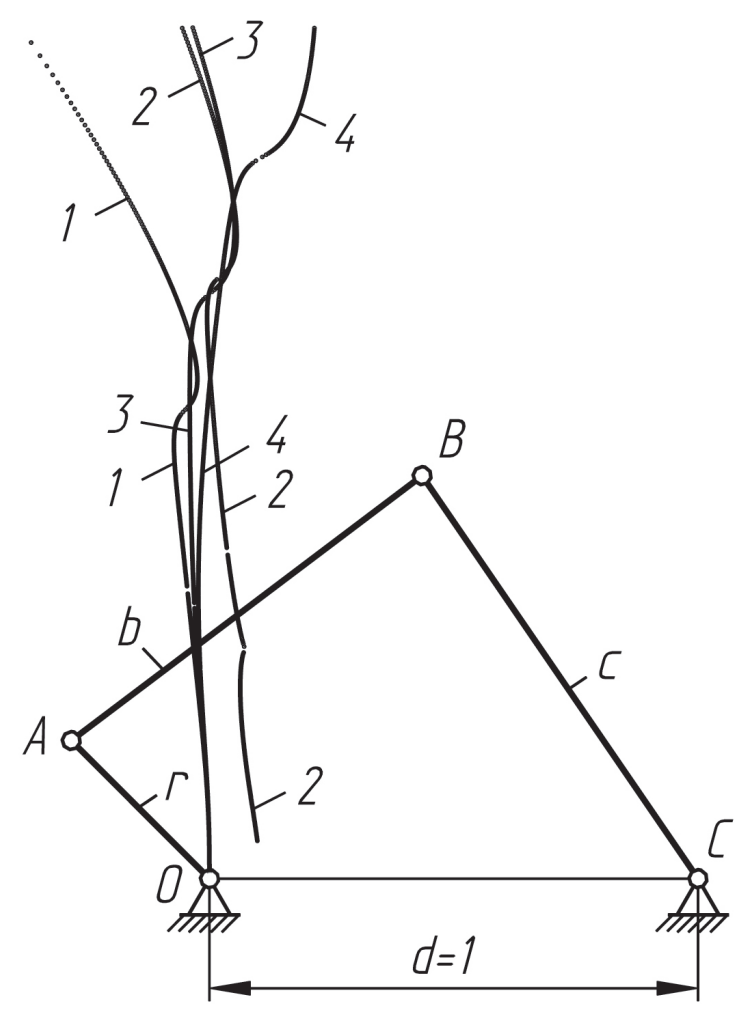

Fig. 3. Samples of the curves of Ball-Burmester points (fivefold interpolation nodes) for the following mechanisms: $1: \mathrm{b}=0.7 ; \mathrm{c}=1.0 ; 2: \mathrm{b}=0.8 ; \mathrm{c}=0.9 ; 3: \mathrm{b}=0.8 ; \mathrm{c}=1.0 ; 4: \mathrm{b}=0.9 ; \mathrm{c}=1,0$. $a$ - the whole view of the curves; $b$-zoomed elements of the curves (view A)

nodes are known [8,9 etc.], but the theoretical grounds of the five infinitesimally close positions of the coupler plane are used only for the synthesis of straight-line linkages, and the length of the straight-line part of the obtained coupler curve cannot be predicted analytically. Thus, the special points of the coupler plane, including a Ball-Burmester point, may define the dwell mechanisms a)

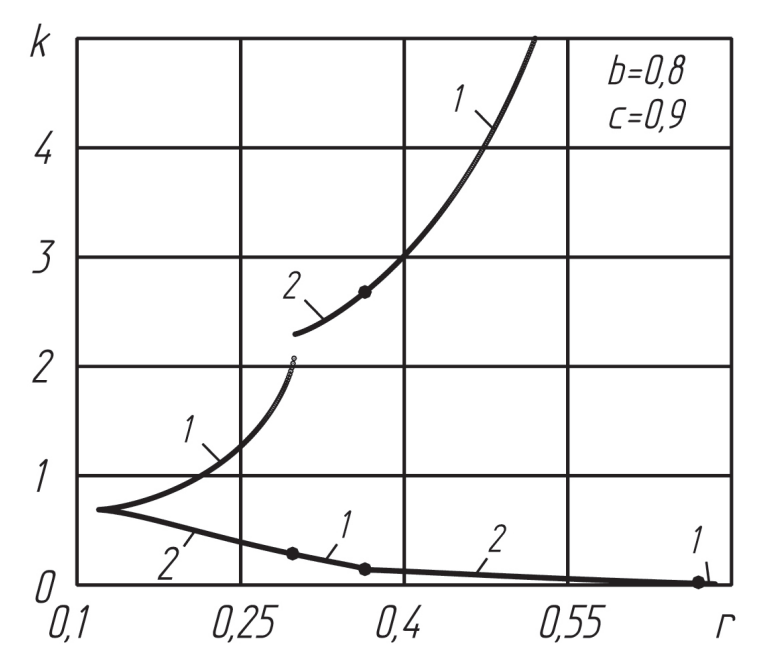

b)

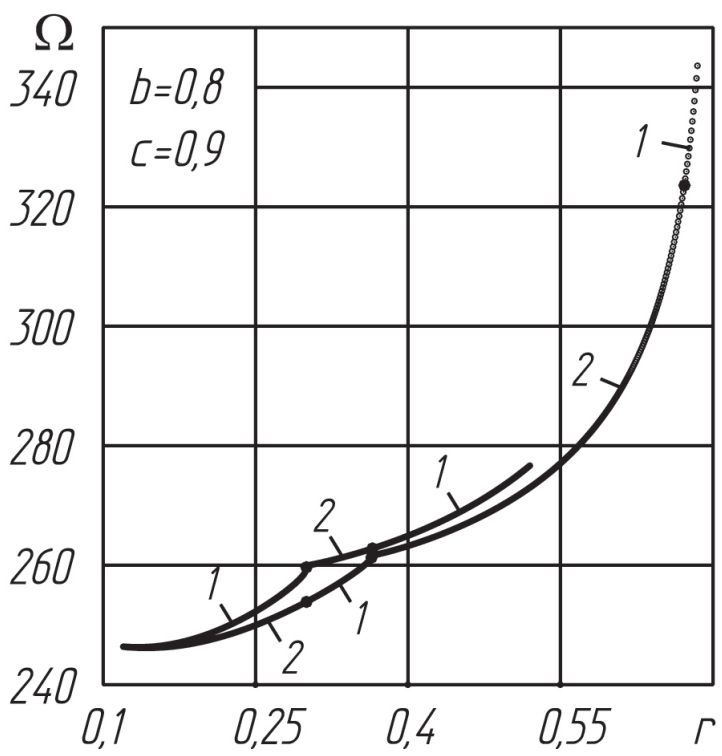

Fig. 4. Diagrams for the determination of the parameters of the coupler's point $T$ (1,2 - for the first and the second special points that can be found in the coupler plane) 


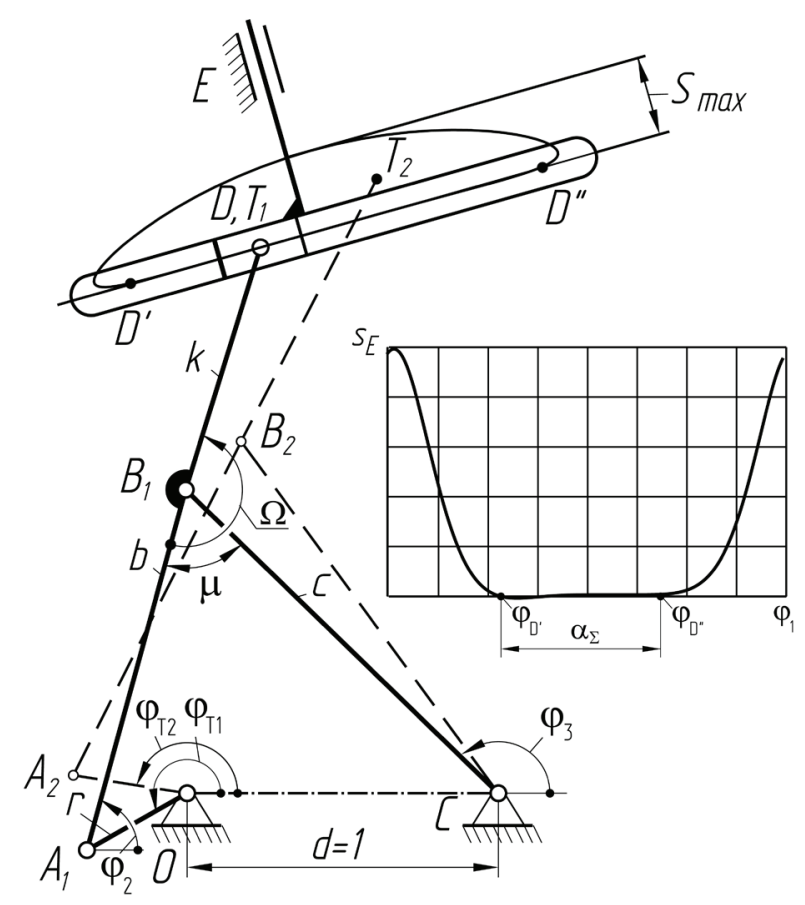

Fig. 5. The six-linked dwell mechanism on the basis of straight-line linkage $O A_{1} B_{1} C D$ with the fivefold interpolation node $T_{1}$, with the diagram of the displacement of the output link $E$

with the different dwell values, and the problem is that it is impossible to predict the dwell value and other parameters of the mechanism using only the analytical methods of kinematic geometry.

Therefore, the unsolved task is to synthesize the dwell mechanisms by the given duration of the dwell and other design parameters that are important for the designer. In order to determine a dwell time of the output link, a numerical method that is based on the usage of unitless coefficient of maximum velocity can be used, as it is proposed in the work [3]. We also used this method in the work [4] to carry out the synthesis procedure of the dwell linkages that are based on Burmester points. The obtained results proved the correctness of the proposed method. Since the Ball-Burmester points hold the condition of $4^{\text {th }}$ order of tangency to the tangent line, they should theoretically define mechanisms with long dwells and high accuracy. Fig. 6 shows the examples of the obtained results.

The calculations were carried out for the different combinations of the lengths of the basic four-bar linkage $(r, b, c)$, and it was established a)

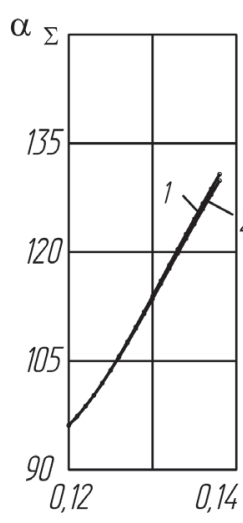

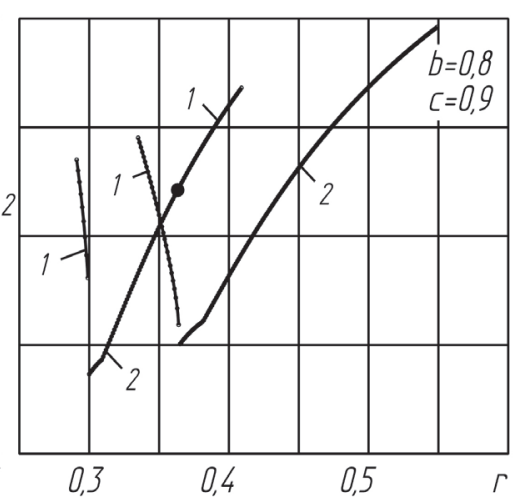

b)

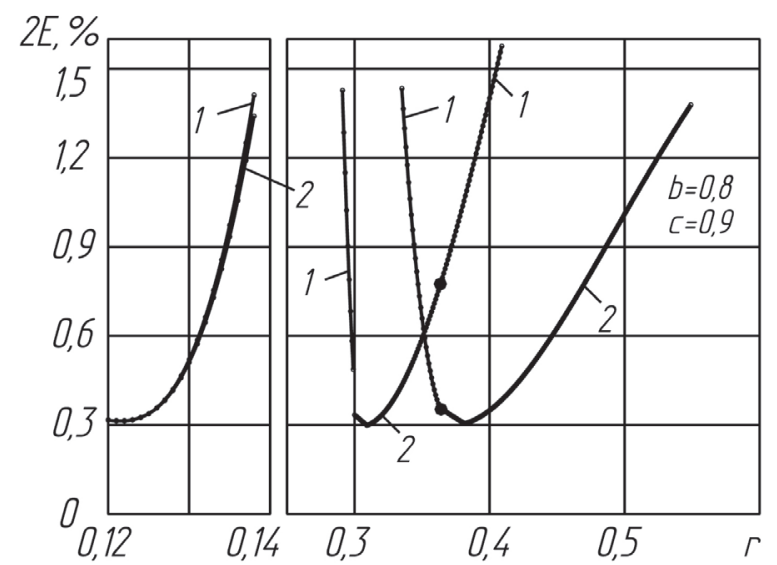

Fig. 6. Diagrams for the parameters of the dwell: $a$-dwell value; $b$ - maximum deviation from the straight line of the approximated part (percentage from the maximum displacement $S_{\max }$ of the output link) 1,2 - diagrams for the first $T_{1}$ and the second $T_{2}$ special points 
that the majority of mechanisms that are synthesized on the basis of the Ball-Burmester points have long dwells within the range $\alpha_{\Sigma}=90^{\circ}-160^{\circ}$, and the accuracy of the approximation $2 E$ is higher in comparison to the mechanisms that can be synthesized using other methods [3,9]. It was also established that not all the special points of the Ball-Burmester points' curves (Fig. 3), which are defined by the parameters $k$ and $\Omega$ (Fig. 4), are suitable for the practical usage. For the recommended conditions of the force transmission in the mechanism and to prevent the self-locking

a)

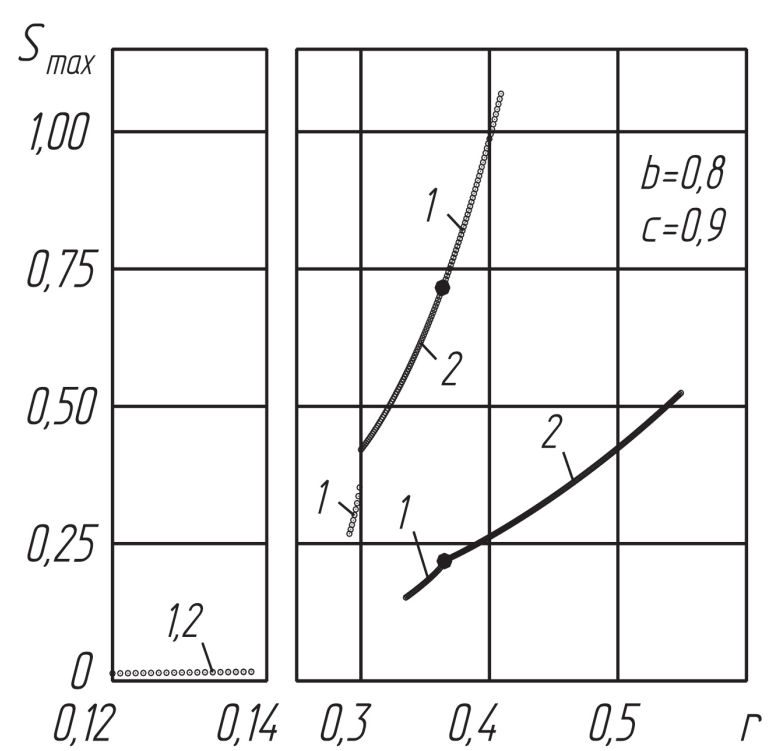

c)

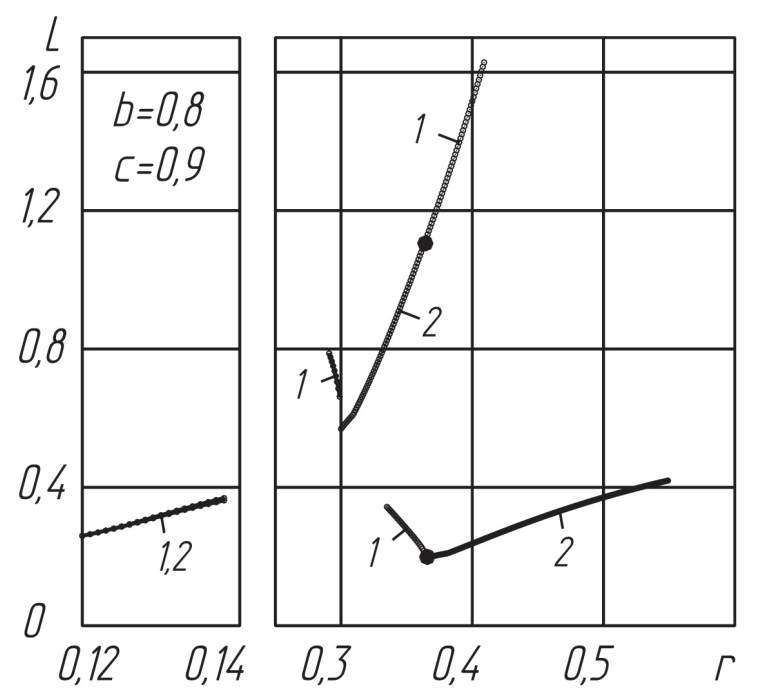

of the links, the transmission angle $\mu$ (see Fig. 5) should be within the following range [3]:

$$
30^{\circ} \leq \mu \leq 150^{\circ} \text {. }
$$

The obtained diagram of the transmission angles of the straight-line linkages with fivefold interpolation nodes is shown in the Fig. 7, $d$. Therefore, the results in Fig. 6 and Fig.7, $a-c$ are shown only for the mechanisms, in which the dimensions of the links hold the condition (3). Besides, the authors considered only the mechanisms with the dwell of the output link in one of the extreme

b)

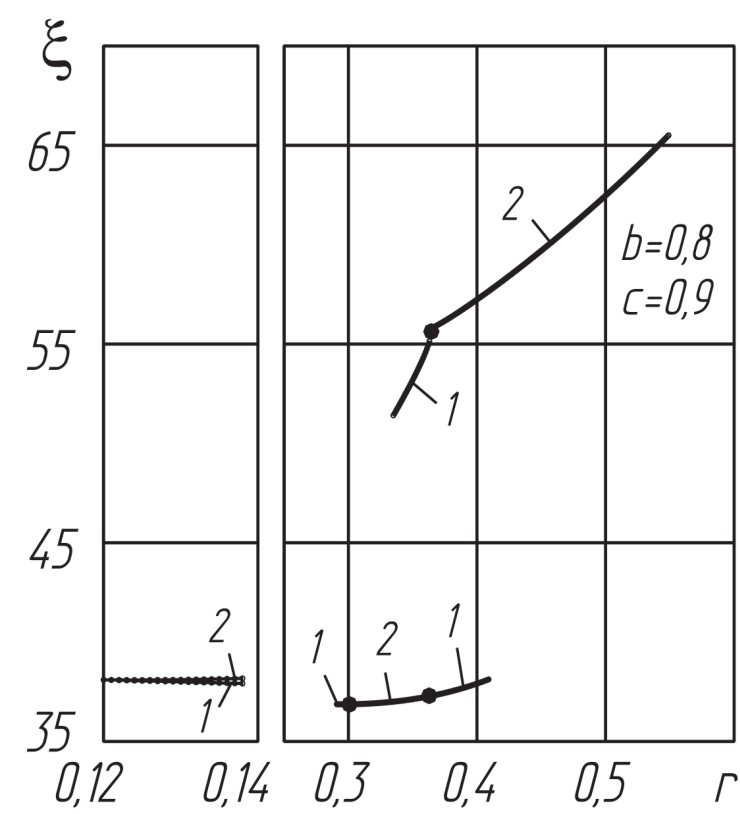

d)

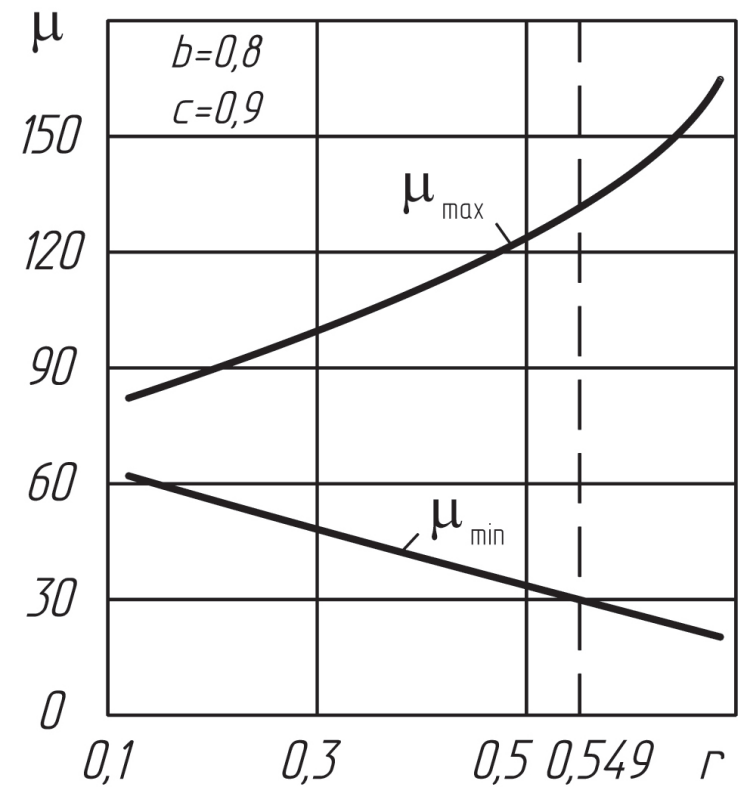

Fig. 7. Diagrams for the determination of the parameters of the basic straight-line and dwell linkages for the first $T_{1}$ and the second $T_{2}$ special points 
positions (as shown in Fig. 5), the mechanisms with the dwells inside the displacement of the output link were eliminated.

The important parameter of the dwell mechanism is the necessary maximum displacement $S_{\max }$ of the output link that is often given by the designer and is defined by the technological application of the machine (Fig. 7, a). Besides, it is important for the designer to take into account the angle of inclination $\xi$ of the straight-line part, which defines the overall design of the mechanism (Fig. 7,b). As it is known, straight-line linkages can be used not only as a basic part of dwell mechanisms, but also have an independent application in machines. In that case, it is an important task to synthesize those mechanisms by the given length $L$ of the straight-line (Fig. 7, c).

\section{CONCLUSIONS}

The results of the kinematic synthesis of dwell mechanisms that are shown above are the samples that were obtained by the developed algorithms and software. They show the capabilities of the dwell mechanisms that are designed using the Ball-Burmester points (fivefold interpolation nodes) by the analytical kinematic geometry methods with the numerical optimization procedures. The reference diagrams that are shown in the paper can be built for any initial values of the mechanism parameters, it helps the designer to define the parameters of the mechanism at the first stage of design, in order to define the areas of mechanisms existence. It is also possible to carry out an optimization procedure by different criteria.

The received results showed that the dwell linkages on the basis on the fivefold interpolation nodes can provide large dwells with high accuracy of approximation. Thus, it enables to use linkage mechanisms instead of the ones with the higher kinematic pairs, it is important because linkages have a number of essential practical advantages over other types of mechanisms. Further research is planned to be carried out in the field of optimization by the main kinematic parameters of the synthesized dwell linkage mechanisms.

\section{REFERENCES}

1. Hricko J. Straight-line mechanisms as one building element of small precise robotic devices. Applied Mechanics and Materials, 613, 2014, 96-101.

2. McCarthy J., Soh G., Geometric Design of Linkages, 2nd edition. Springer-Verlag, New York, 2011, pp. 448.

3. Kharzhevskyi V.O. Synthesis of the linkage mechanisms with dwell of the output links using kinematic geometry methods: monography. Khmelnytskyi RVC KhNU, 2015 (in Ukrainian).

4. Kharzhevskyi V.O. Kinematic synthesis of linkage mechanisms using Burmester points at the given dwell duration of the output link. Advances in Science and Technology Research Journal, 2(11), 2017, 139-145.

5. Norton R.L. Design of Machinery: An Introduction to the Synthesis and Analysis of Mechanisms and Machines. McGraw Hill, 2011.

6. Pashechko M., Pasika V., Hembara N., Kharzhevskyi V. Analysis of Linkage Mechanisms with Internal Driving Link. Advances in Science and Technology Research Journal, 1(14), 2020, 191-200.

7. Sarkissyan Y.L. Approximations in Synthesis of Mechanisms. State Engineering University of Armenia Proceedings, Series "Mechanics, Machine Science, Machine-building”, 15(2), 2012, 9-21.

8. Yin L., Han J., Huang J., Yang T., Yin L. A General Method for Synthesizing Straight-Line Linkage with Ball and Burmester Points. Applied Mechanics and Materials, 215-216, 2012, 138-141.

9. Wang D. Wang W. Kinematic Differential Geometry and Saddle Synthesis of Linkages. John Wiley \& Sons Singapore Pte. Ltd., 2015, pp. 450. 